\title{
OPTICAL PDMS MICROFIBRE SENSOR FOR DISPLACEMENT DETERMINATION
}

We prepared and demonstrated the usage of an optical polydimethylsiloxane (PDMS) microfibre sensor for displacement measurement. The sensor consists of PDMS microfibre placed between two conventional single-mode optical fibres. For proper bend of the microfibre the structure forms Mach-Zehnder interferometer. In that case, one arm of the interferometer consists of the microfibre and second one of the air. In addition, it is possible to resize the air arm in a range of several tens of micrometres with sufficient ratio of intensities between lights transmitted through the interferometer arms. For optical paths difference smaller than optical coherence of light source one can observe the interference of light. Determination of the displacement was done based on the spectral shift of interference pattern for particular lengths of air arm. The sensitivity of the sensor was $2.5 \mathrm{~nm} / \mu \mathrm{m}$.

Keywords: Microfiber, PDMS, displacement determination, optical sensor, Mach-Zehnder interferometer.

\section{Introduction}

Optical devices based on optical microfibres can be used in a wide range of applications for their unique properties such as large evanescent field and strong confinement of the propagated light [1]. Moreover, the optical microfibres can be arranged in different configurations such as taper [2], knot resonator [3], part of interferometers $[2,4,5]$ and made from different materials such as silica [6, 7], polysiloxanes [5]. This diversity predisposes them to sensing various physical quantities, for example temperature [7, 8], strain [7, 8], refractive index [9], volatile organic compounds $[1,10]$, current $[4]$ and displacement $[2,11]$.

In the paper, we deal with the process of microfibre fabrication from polydimethylsiloxane (PDMS) and its usage for displacement determination. By proper bending of optical microfibre the Mach-Zehnder interferometer is formed, one arm consists of microfibre and the second one of air. Since the optical path difference is smaller than coherence length of optical source the interference of beams is observed. We are focused on the displacement determination by means of determining wavelength shifts of interference minima in frequency domain.

\section{Optical microfibre fabrication}

For preparation of optical microfibre integrated between two conventional single-mode optical fibres (SMFs) we used liquid silicone Sylgard 184 (Dow Corning) supplied as two-part liquid component kits. The silicone is highly light-transparent material, chemically inert, thermally stable, permeable to gasses, simple to handle, elastically deformable to a large extent and has a great potential for photonics [12]. After mixing the pre-polymer and curing agent at ratio of 10:1, the prepared elastomer was applied on the cleaved ends of conventional optical fibres (Fig. 1a). Thereafter, we connected optical fibre ends covered by PDMS (Fig. 1b). Then PDMS was cured at room temperature for about 10 hours. After that time the PDMS elastomer achieved suitable consistency for microfibre drawing. Axial alignment of SMFs with PDMS joint was mechanically adjusted by $3 \mathrm{D}$ microstages a)
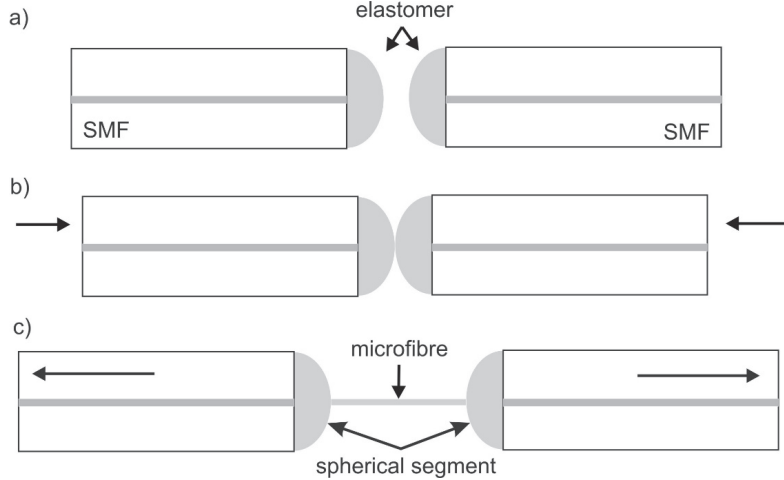

Fig. 1 Illustration of optical microfibre fabrication: a) PDMS deposition on single-mode fibre (SMF) ends, b) connection of fibre ends.

c) coaxial drawing

\footnotetext{
* Daniel Kacik, Ivan Martincek, Norbert Tarjanyi

Department of Physics, Faculty of Electrical Engineering, University of Zilina, Slovakia

E-mail: daniel.kacik@fel.uniza.sk
} 
to obtain maximal transmitted signal through the PDMS joint at wavelength $1550 \mathrm{~nm}$. PDMS microfibre was created by gradual distancing of single-mode fibres ends (Fig. 1c) together with in-situ controlling the axial position by signal level. When the length and the diameter of microfibre was sufficient the PDMS microfiber was cured at the room temperature for another 40 hours. The prepared optical microfibre had a diameter of 7 micrometres and 270 micrometres in length. This microfiber was formed on the PDMS spherical segments with height of about 50 micrometres placed at the end faces of SMFs.

\section{Mach-Zehnder interferometer}

The light transmitted through the optical fibre is coupled to the PDMS spherical segment and then to the optical microfibre. After passing the PDMS microfibre the light is recoupled to the output optical fibre and propagates there. But for suitable bend of optical microfibre (the bend of microfibre was done by decreasing the distance between ends of SMFs) it is possible to obtain the state when the light transmitted through the SMF is split at the spherical segment placed on the end of optical fibre: the part of light is still transmitted through the microfibre and another part is propagated in coaxial direction to core of input fibre (so the light propagates in the air path). By proper adjusting of output fibre position it is possible to recouple the light transmitted through the air path to the output fibre (enlargement in Fig. 2).

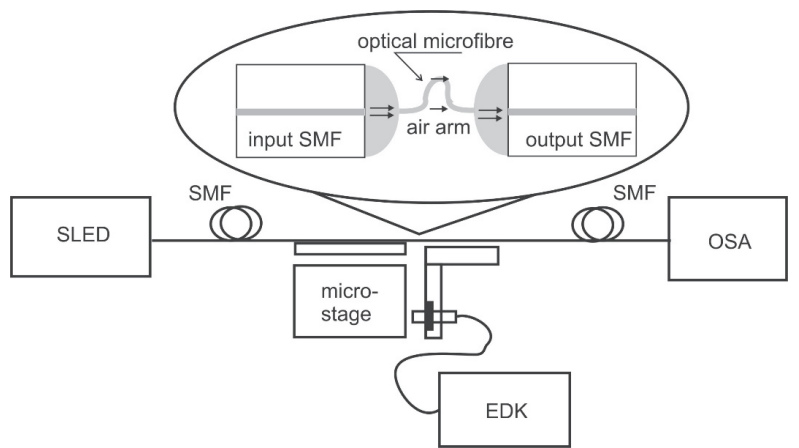

Fig. 2 Experimental setup with detail of sensor: SLED - optical source, EDK - commercial displacement meter with inductance probe,

OSA - optical spectrum analyser

If the optical path difference (between light transmitted through the microfibre and air path) is smaller than coherence length of an optical source one can observe the interference of light.

In this case, the interference pattern is formed by two beams, one guided through the microfibre and second one through the air path. The output intensity can be expressed as

$$
I(\lambda)=I_{1}+I_{2}+2 \sqrt{I_{1} I_{2}} \cos \left(\beta(\lambda) z_{1}-\frac{2 \pi n}{\lambda} z_{2}\right)
$$

where $I_{1}$ and $I_{2}$ are intensities of light coupled to the core of the output SMF from the microfibre and from the air path, respectively, $\beta$ is phase constant of the beam (mode) guided through the microfibre, $z_{1}$ is the length of the microfibre, $z_{2}$ is length of air path, $n$ is refractive index of air and $\lambda$ is the wavelength of the propagating light in vacuum.

\section{Displacement sensing}

For investigation of spectral dependence of interference pattern of the prepared PDMS microfibre sensor for the displacement the broadband light source coupled to the optical fibre (SLED Safibra OFLS-6) with central wavelength at 1550 $\mathrm{nm}$ and FWHM of $62 \mathrm{~nm}$ was used. The output SMF was connected to and the spectrum recorded by an optical spectrum analyser (Anritsu MS9710B), with a resolution of $0.07 \mathrm{~nm}$. The sensor consisting from SMFs ends covered by PDMS and optical microfibre was placed on differential micrometre stages to adjust the relative positions of SMFs and resizing the length of the air arm. The length of air arm was measured by commercial displacement meter with inductance probe, which allows one to distinguish variations of the length with resolution of hundredth of a micrometre. Schematic of experimental setup is shown in Fig. 2.

The transmission spectra of prepared PDMS microfibre sensor in the wavelength range from $1545 \mathrm{~nm}$ to $1565 \mathrm{~nm}$ (it was measured in wavelength range $1500 \mathrm{~nm}$ to $1600 \mathrm{~nm}$ but for better illustration was chosen only narrow interval) at constant room temperature and atmospheric pressure for resizing length of the air arm in the interval $0 \mu \mathrm{m}$ to $4 \mu \mathrm{m}$ with step $1 \mu \mathrm{m}$ are shown in Fig. 3. As it can be seen from dependencies shown in Fig. 3 for particular length of the air arm the power level is very slightly changed. It could be caused by the change of the intensity of light recoupled to the output fibre due to the change of the microfibre bend or misalignment of the output fibre. Nevertheless for the evaluation of the measurement this can be neglected.

In paper [11] we investigated the effect of the air arm length on the period of interference pattern. The change in the period of interference pattern of the structure was determined to 0.027 $\mathrm{nm}$ per micrometre. For a small displacement (and for resolution of optical spectrum analyser) we could not use this approach. So for determination of such a small displacement we investigated the wavelength shifts of the interference pattern. We choose the minima of the interference pattern and determined its wavelength (reference). For the particular displacement determined by displacement meter, the wavelength shift of reference minima of the interference pattern was observed (see Fig. 3).

The dependence of measured wavelength shift $\Delta \lambda_{\mathrm{i}}$ on the displacement and its linear fit are shown in Fig. 4. The sensitivity of sensor $2.5 \mathrm{~nm}$ per micrometre can be determined from the linear fit. According to [11], it has almost 100 times higher 


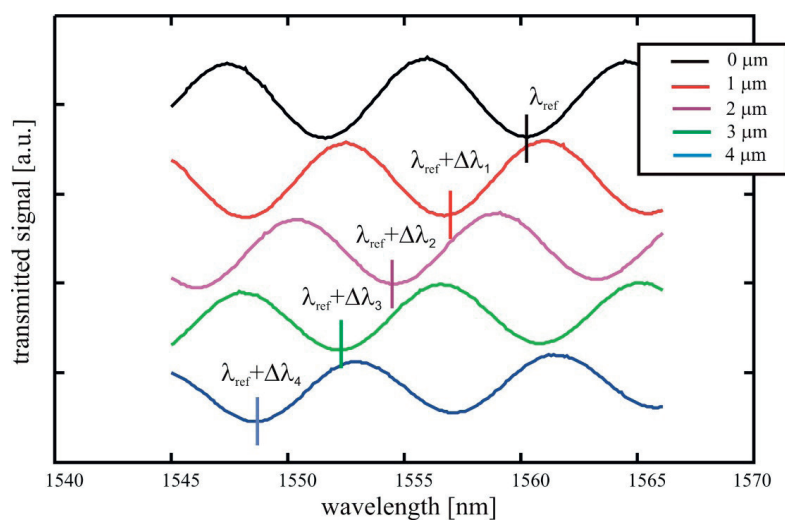

Fig. 3 Vertical shifted transmission spectra of interferometer for different measured air arm lengths; there are also indicated wavelength of reference minima $\lambda_{\text {ref }}$ and its particular shift $\Delta \lambda$ for particular displacement

sensitivity. But disadvantage of the evaluation is that after crossing phase change by $2 \pi$ without observation of position of the interference minima it is not possible to determine the value of displacement. So it is possible to determine it only when the displacement is up to $3.14 \mu \mathrm{m}$ (corresponds to $8.6 \mathrm{~nm}$ wavelength shift).

\section{Conclusion}

We have demonstrated the preparation of an optical PDMS microfibre which is directly integrated between single-mode optical fibres, the transmission properties of the prepared sensor and its application in the displacement determination by the measurement of spectral shifts of minima of interference pattern.

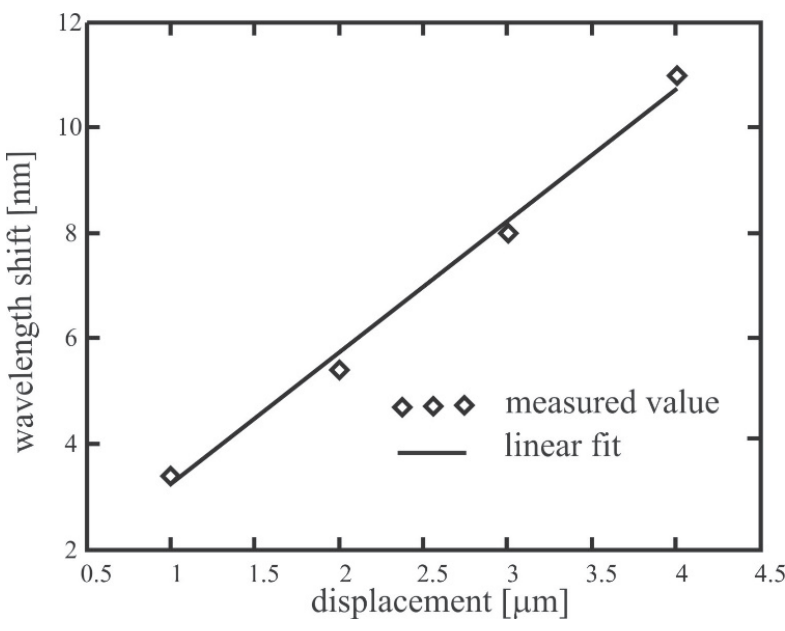

Fig. 4 The dependence of wavelength shift on displacement

The prepared microfibre sensor is a two-arm interferometer in which one arm consists of a bend PDMS microfibre and the second is formed by an air line. We measured the displacement experimentally to the value of 4 micrometres, with an accuracy better than $\pm 0.01 \mu \mathrm{m}$. The sensitivity in this range was determined to $2.5 \mathrm{~nm} / \mu \mathrm{m}$. For the optical PDMS microfibre the way of displacement determination can be used to the value of $3.14 \mu \mathrm{m}$ due to phase of interference pattern is changed over $2 \pi$.

\section{Acknowledgements}

This work was supported by Slovak National Grant Agency No. VEGA 1/0491/14, 1/0278/15 and Slovak Research and Development Agency under the project No. APVV-15-0441.

\section{References}

[1] LUO, J., WANG, Y., TONG, L.: Microfiber Optical Sensors: A Review. Sensors 14(4), 5823-5844, 2014.

[2] LUO, H., LI, X., ZOU, W., LI, X., HONG, Z., CHEN, J.: Temperature-Insensitive Microdisplacement Sensor Based on Locally Bent Microfiber Taper Modal Interferometer. IEEE Photonics Journal, 4(3), 772-778, 2012.

[3] GOMES, A. D., FRAZAO, O.: Microfiber Knot Resonator as Sensor - A Review., Proc. of $5^{\text {th }}$ intern. Conference on Photonics, Optics and Laser Technology (PHOTOPTICS 2017), Portugal, 356-364, 2017.

[4] JASIM, A. A., HARUN, S. W., MUHAMMAD, M. Z., AROF, H., AHMAD, H.: Current Sensor Based on Inline Microfiber MachZehnder Interferometer. Sensors and Actuators A, 192, 9-12, 2013.

[5] MARTINCEK, I.: Fabrication and Characterisation of Polysiloxane Optical Fiber Microloop Interferometers. Microwave and Optical Technology Letters, 58(12), 2876-2879, 2016.

[6] LOU, J., TONG, L., YE, Z.: Modeling of Silica Nanowires for Optical Sensing. Optics Express, 13(6), 2135-2140, 2005.

[7] MUHAMMAD, M. Z., JASIM, A. A., AHMAD, H., AROF, H., HARUN, S. W.: Non-Adiabatic Silica Microfiber for Strain and Temperature Sensors. Sensors and Actuators A, 192, 130-132, 2013.

[8] TIAN, J., LIU, S., YU, W., DENG, P.: Microfiber Bragg Grating for Temperature and Strain Sensing Applications. Photonic Sensors, 7(1), 44-47, 2017. 
[9] LUO, H., SUN, Q., XU, Z., JIA, W., LIU, D., ZHANG, L.: Microfiber-Based Inline Mach Zehnder Interferometer for DualParameter Measurement. IEEE Photonics Journal, 7(2), 7100908, 2015.

[10] KACIK, D., MARTINCEK, I.: Toluene Optical Fibre Sensor Based on Air Microcavity in PDMS. Optical Fiber Technology, 34, 70-73, 2017

[11] KACIK, D., MARTINCEK, I.: Optical Structure with PDMS Microfibre for Displacement Measurement. Proc. of $5^{\text {th }}$ intern. Conference on Photonics, Optics and Laser Technology (PHOTOPTICS 2017), Portugal, 365-368, 2017.

[12] PUDIS, D., JANDURA, D., GASO, P., SUSLIK, L., HRONEC, P., MARTINCEK, I., KOVAC, J., BEREZINA, S.: PDMS-based Nanoimprint Lithography for Photonics. Communications - Scientific Letters of the University of Zilina, 16(1), 15-20, 2014. 